\title{
Treating Patients with Type 2 Diabetes Mellitus Uncontrolled on Basal Insulin in the Czech Republic: Cost-Effectiveness of IDegLira Versus iGlarLixi
}

\author{
Johannes Pöhlmann (D) · Monika Russel-Szymczyk · Pavel Holík •
}

Karel Rychna · Barnaby Hunt (D)

Received: December 11, 2018 / Published online: January 31, 2019

(C) The Author(s) 2019

\begin{abstract}
Introduction: Few patients with type 2 diabetes mellitus (T2DM) achieve recommended glycemic control targets in the Czech Republic. Novel therapies, such as fixed-ratio combinations of basal insulin plus glucagon-like peptide-1 receptor agonists, may contribute to better glycemic control. In the analysis presented here, the present analysis assessed the long-term cost-effectiveness of two fixed-ratio combinations, IDegLira (insulin degludec/liraglutide) and iGlarLixi (insulin glargine/lixisenatide), for the treatment of patients with T2DM inadequately controlled with basal insulin from a healthcare payer perspective in the Czech Republic.
\end{abstract}

Enhanced Digital Features To view enhanced digital features for this article go to: https://doi.org/10.6084/ m9.figshare.7583651.

Electronic Supplementary Material The online version of this article (https://doi.org/10.1007/s13300019-0569-7) contains supplementary material, which is available to authorized users.

J. Pöhlmann $(\varangle) \cdot$ B. Hunt

Ossian Health Economics and Communications, Basel, Switzerland

e-mail: poehlmann@ossianconsulting.com

M. Russel-Szymczyk

Novo Nordisk Pharma Sp. z.o.o., Warsaw, Poland

P. Holík · K. Rychna

Novo Nordisk s.r.o., Prague, Czech Republic
Methods: A cost-effectiveness analysis was performed over patient lifetimes using the IQVIA CORE Diabetes Model. Treatment effects were obtained from an indirect treatment comparison as no head-to-head data for IDegLira versus iGlarLixi are currently available. IDegLira was compared with two iGlarLixi pens $(100 \mathrm{U} / \mathrm{mL}$ insulin glargine $+33 \mu \mathrm{g} / \mathrm{mL}$ and $50 \mu \mathrm{g} / \mathrm{mL}$ of lixisenatide, respectively). Direct medical costs associated with pharmaceutical interventions, screening and diabetes-related complications were captured. Deterministic and probabilistic sensitivity analyses were performed.

Results: IDegLira was associated with gains in life expectancy of 0.11 years and in quality-adjusted life expectancy of 0.14 quality-adjusted life-years (QALYs) versus iGlarLixi, due to a lower cumulative incidence and delayed onset of diabetes-related complications. IDegLira was also associated with higher projected costs due to higher acquisition costs; however, these were partially offset by cost savings from avoided complications. IDegLira was associated with incremental cost-effectiveness ratios of Czech Koruna (CZK) 695,998 and CZK 348,323 per QALY gained versus iGlarLixi pens containing 33 and $50 \mu \mathrm{g} / \mathrm{mL}$ of lixisenatide, respectively. These ratios were below the commonly used willingness-to-pay threshold of CZK 1,200,000 per QALY gained.

Conclusion: The present analysis indicated that IDegLira was associated with clinical benefits relative to iGlarLixi over patient lifetimes and 
was likely to be cost-effective in the treatment of patients with T2DM uncontrolled on basal insulin in the Czech Republic.

Funding: Novo Nordisk.

Plain Language Summary: Plain language summary is available for this article.

Keywords: Cost-effectiveness; Czech Republic; Fixed-ratio combination; IDegLira; iGlarLixi; Type 2 diabetes

\section{PLAIN LANGUAGE SUMMARY}

- Patients with type 2 diabetes mellitus (T2DM) benefit from reductions in blood sugar levels and body weight, which lower the risk of long-term diabetes-related complications. Novel treatments, such as fixedratio combinations (FRCs) of insulin plus glucagon-like peptide-1 receptor agonists, can help patients to achieve these treatment targets, at low risk of hypoglycemia.

- In the Czech Republic, too few patients with T2DM achieve treatment targets, and diabetes imposes a substantial cost burden on the healthcare system. Modern antidiabetic treatments, such as FRCs (e.g. IDegLira and iGlarLixi), provide the means to improve diabetes treatment and reduce diabetes-related costs. As healthcare budgets are not limitless, healthcare payers need to choose cost-effective treatments to achieve the best possible use of budgets. The present study evaluated the long-term cost-effectiveness of IDegLira versus iGlarLixi in Czech patients with T2DM poorly controlled on basal insulin.

- A recent network meta-analysis (NMA) comparing IDegLira and iGlarLixi reported reductions in blood sugar levels (measured as glycated hemoglobin) and body weight, as well as lower hypoglycemia rates, for IDegLira relative to iGlarLixi. As no headto-head studies comparing the two FRCs are available, the NMA is the best source to inform long-term modeling.

- Relative to iGlarLixi, IDegLira was associated with higher life expectancy and qualityadjusted life expectancy. Over patient lifetimes, the costs of diabetes-related complications were lower in patients treated with IDegLira and partly offset the higher acquisition costs of IDegLira.

- In the Czech Republic, IDegLira is a costeffective alternative to iGlarLixi for the treatment of patients with T2DM poorly controlled on basal insulin.

\section{INTRODUCTION}

Diabetes is considered a "global pandemic" of the twenty-first century and associated with a substantial clinical and economic burden on patients and healthcare systems [1]. In 2017, it was estimated that 863,106 people in the Czech Republic were living with diagnosed diabetes (patients with impaired glucose tolerance not included), of whom $84 \%$ had type 2 diabetes mellitus (T2DM) [2]. Good glycemic control is crucial to reduce the incidence of diabetes-related complications and, consequently, the clinical and economic burden associated with diabetes [3, 4]. In the Czech Republic, lifestyle changes and metformin therapy are recommended as first-line therapy for patients with T2DM [5]. If glycated hemoglobin (HbA1c) levels do not fall below 7.0\% within 6 months while on this therapeutic regimen, then intensification to dual therapy with other non-insulin antidiabetic medications (including glucagon-like peptide-1 [GLP-1] receptor agonists) or insulin is recommended. If the $\mathrm{HbA} 1 \mathrm{c}$ target is then not reached within another 6 months, treatment with an intensive insulin regimen or combination therapy (of non-insulin antidiabetic medications) is recommended to achieve a target HbA1c level of $7.0 \%$ [5]. In the Czech Republic, however, only about onethird of patients with T2DM achieve an HbA1c target of $7.0 \%$, as recently demonstrated in the DIAINFORM study [6]. No measurable improvements in glycemic control were identified over the 3 years prior to the study, and clinical inertia was considered by the authors to be a likely cause for the lack of progress, leading them to call for the use of novel antidiabetic therapies [6]. 
Fixed-ratio combinations of GLP-1 receptor agonists plus basal insulin represent such novel treatments for patients with T2DM who fail to achieve adequate glycemic control. These combinations could be attractive treatment options as they combine the complementary effects of their components $[7,8]$. While basal insulin provides a stable, long-acting reduction in HbA1c levels, GLP-1 receptor agonists stimulate insulin secretion and reduce hepatic glucose production in a glucose-dependent manner, thereby improving glucose control, particularly of post-prandial glucose levels, with a low risk of hypoglycemia [9]. In addition, compared with basal insulins which often lead to gains in body weight, fixed-ratio combinations are generally associated with reduced body weight in populations poorly controlled on basal insulin or oral antidiabetic therapy $[7,8]$.

The first fixed-ratio combination to be introduced to the European market was IDegLira (Xultophy®; Novo Nordisk, Bagsværd, Denmark), which is a combination of insulin degludec (IDeg) and liraglutide [7, 9]. IDegLira was demonstrated in the Dual Action of Liraglutide and Insulin Degludec in Type 2 Diabetes (DUAL) trial program to be associated with improved glycemic control in a wide range of patients, including patients treated with pioglitazone, sulfonylureas, oral antidiabetics (OAD), GLP-1 receptor agonists and basal insulin [10-14]. Importantly, reductions in HbA1c were associated with reduced glycemic variability and achieved without weight gain and at low risk of hypoglycemia [10, 13-15]. More recently, iGlarLixi (Suliqua ${ }^{\circledR}$; Sanofi S.A., Paris, France), a fixed-ratio combination of insulin glargine (IGlar) plus lixisenatide, became available in the Czech Republic. In the LixiLan trial program, iGlarLixi was shown to be associated with larger HbA1c reductions than IGlar in patients with T2DM inadequately controlled on OAD therapy and basal insulin plus metformin, with fewer gastrointestinal side effects than with lixisenatide alone $[16,17]$.

Both IDegLira and iGlarLixi are reimbursed in the Czech Republic for adult patients with T2DM inadequately controlled on basal insulin. Given the differences in the clinical and cost profiles of the two drugs, a cost-effectiveness analysis was conducted to inform resource allocation within budget constraints of healthcare systems [18, 19]. Cost-effectiveness analysis and budget impact analysis represent the two key assessments required for a drug to be reimbursed in the Czech Republic. Therefore, the aim of the analysis reported here was to assess the long-term cost-effectiveness of IDegLira versus iGlarLixi for the treatment of patients with T2DM inadequately controlled on basal insulin, from a healthcare payer perspective in the Czech Republic.

\section{METHODS}

\section{Choice of Comparator}

Before iGlarLixi became available in the Czech Republic, comparators for IDegLira were limited to basal-bolus insulin regimens and combination therapies, such as GLP-1 receptor agonists plus basal insulin, which have been included in an earlier economic evaluation versus IDegLira $[5,20]$. Since receiving its marketing authorization in 2017, iGlarLixi has been considered the most relevant comparator for IDegLira. As the two therapies have not previously been assessed with regard to their relative cost-effectiveness in the Czech Republic, iGlarLixi was chosen as the comparator in the present analysis.

In the Czech Republic, iGlarLixi is available in pre-filled pens in two strengths: a pen combining $100 \mathrm{U} / \mathrm{mL}$ of IGlar with $33 \mu \mathrm{g} / \mathrm{mL}$ of lixisenatide (providing daily doses of 30-60 dose-steps) and a pen combining $100 \mathrm{U} / \mathrm{mL}$ of IGlar with $50 \mu \mathrm{g} / \mathrm{mL}$ of lixisenatide (providing daily doses of 10-40 dose-steps). As acquisition costs differ between pens, both pens were included in the present cost-effectiveness analysis.

\section{Description of the Modeling Approach}

The cost-effectiveness of IDegLira versus iGlarLixi was evaluated by projecting long-term cost and health outcomes for both treatments. Costs were expressed in monetary units (Czech 
Koruna [CZK]) and health outcomes in qualityadjusted life-years (QALYs). Cost-effectiveness was expressed as an incremental cost-effectiveness ratio (ICER), i.e. the difference in projected costs divided by the difference in projected health outcomes. The ICER, which was reported as CZK per QALY gained, was compared to a willingness-to-pay (WTP) threshold to assess if IDegLira could be considered to provide good value for money. In the Czech Republic, the commonly accepted WTP threshold is the gross domestic product per capita multiplied by three, as suggested by the World Health Organization [20-23]. In 2018, this value was CZK 1,200,000 (per QALY gained), which was used in this analysis as the WTP threshold.

Long-term estimates of cost and health outcomes associated with each treatment were obtained from simulations using the IQVIA CORE Diabetes Model (CDM; IQVIA, Basel, Switzerland). The CDM is a web-based, nonproduct-specific, interactive computer model developed to project the long-term health and economic outcomes associated with antidiabetic treatment interventions [24, 25]. The model and its validations against real-life data have been described in detail elsewhere [24-26]. Briefly, a series of interdependent sub-models is used to simulate background mortality and diabetes-related complications and assess their impact on quality of life and costs over time. The model can, therefore, be used to extrapolate short-term results, such as those from a clinical trial, to long-term outcomes regarding life expectancy, quality-adjusted life expectancy (QALE), cumulative incidence and time to onset of diabetes-related complications, as well as direct medical costs.

\section{Time Horizon, Treatment Duration and Discounting}

The base case analysis was performed over patients' lifetime. A long time horizon is recommended to fully capture long-term diabetesrelated complications and their impact on life expectancy, quality of life and costs. Shorter time horizons were explored in sensitivity analyses [27]. The model accounted not only for complication-related mortality but also for Czech Republic-specific background mortality [28].

Patients were assumed to receive IDegLira or iGlarLixi for the first 5 years of the analysis before treatment was intensified to basal-bolus insulin for the remainder of their lifetimes. These assumptions reflect the need for further intensification, required for most patients with T2DM to maintain good long-term glycemic control, and are in line with previous cost-effectiveness analyses of IDegLira in the Czech Republic [20].

As cost and benefits occurring in the future are generally valued differently from cost and benefits occurring in the present, discounting future outcomes is recommended by Czech guidelines for health economic analyses with a time horizon of more than 1 year [21, 29]. As specified by guidelines, a discount rate of $3 \%$ per annum was applied in the base case to both clinical and cost outcomes. In sensitivity analyses, discount rates of 0 and 5\% per annum were used.

\section{Clinical Data: Baseline Characteristics, Treatment Effects and Progression of Physiological Parameters}

Baseline characteristics of the simulated patient cohort were sourced from the IDegLira arm of the 26-week, phase 3 DUAL II trial, in line with previous health economic analyses of IDegLira for the Czech Republic (Table 1) [13, 20]. The average number of cigarettes smoked per day and mean weekly alcohol intake, which were not reported in the DUAL II trial, were obtained from Czech Republic-specific data, assuming that the pattern observed for the general Czech population was applicable to the simulated cohort [30, 31].

Treatment effects for IDegLira were taken from the DUAL II trial (Table 2) [13]. Treatment effects for iGlarLixi were calculated by applying the between-treatment differences (in HbA1c, body weight and daily doses) and rate ratios (in hypoglycemic event rates) obtained from a previously published indirect treatment comparison (ITC) to the treatments effects for 
Table 1 Baseline cohort characteristics

\begin{tabular}{ll}
\hline Demographic/risk factor & $\begin{array}{l}\text { IDegLira arm of the } \\
\text { DUAL II trial [13] }\end{array}$ \\
\hline Age (years) & $56.8(8.9)$ \\
Duration of diabetes (years) & $10.3(6.0)$ \\
Proportion of men (\%) & 56.3 \\
HbAlc (\%) & $8.7(0.70)$ \\
Systolic blood pressure & $132.4(14.8)$ \\
$\quad$ (mmHg) & $182.0(45.5)$ \\
Total cholesterol (mg/dL) & $43.4(11.0)$ \\
HDL cholesterol (mg/dL) & $101.9(37.1)$ \\
LDL cholesterol (mg/dL) & $196.8(148.0)$ \\
Triglycerides (mg/dL) & $33.6(5.70)$ \\
BMI (kg/m $\left.{ }^{2}\right)$ & 16.1 \\
Smokers $(\%)$ & 24.5 \\
Cigarettes per day $(n)^{\mathrm{a}}$ & 8.42 \\
Alcohol consumption & \\
$\quad(\mathrm{fl} \mathrm{oz} /$ week) & \\
\hline
\end{tabular}

Values are presented as the mean with the standard deviation (SD) in parenthesis

$B M I$ Body mass index, DUAL Dual Action of Liraglutide and Insulin Degludec in Type 2 Diabetes, $f$ oz fluid ounce, $H b A 1 c$ glycated hemoglobin, $H D L$ high-density lipoprotein, $L D L$ low-density lipoprotein

a Sourced from Tobacco Atlas data for the Czech Republic [30]

b Sourced from World Health Organization data for the Czech Republic [31]

IDegLira [32]. For example, an HbA1c reduction of $-1.92 \%$ was applied for IDegLira based on results from DUAL II. In the ITC, iGlarLixi was shown to be associated with a mean HbA1c that was $0.44 \%$ higher than that for IDegLira. Consequently, the HbA1c treatment effect for iGlarLixi was calculated as $-1.92 \%+0.44 \%$, yielding an HbA1c reduction of $-1.48 \%$ applied for iGlarLixi in the present analysis (see Table 2 for detailed calculations on how iGlarLixi treatment effects were obtained). The difference in body weight was used to calculate the difference in body mass index (BMI) based on the mean height of patients in the IDegLira arm of DUAL II [13]. This approach to calculate treatment effects was considered the most appropriate approach as no head-to-head study comparing IDegLira and iGlarLixi has yet been conducted. The ITC therefore represents the best currently available evidence for the relative efficacy of the two treatments [33, 34].

Treatment effects for HbA1c and BMI were applied in the first year of the analysis, and differences in $\mathrm{HbA1c}$ and BMI were maintained during fixed-ratio combination treatment in the first 5 years of the analysis. Upon treatment intensification after 5 years, an HbA1c of $7.0 \%$ was assumed in both arms for the remainder of patient lifetimes, in line with glycemic control targets in the Czech Republic [5]. On treatment intensification, BMI was assumed to return to the baseline value. Differences in daily insulin dose or in rates of hypoglycemia were maintained over the first 5 years of the analysis and abolished upon treatment intensification after 5 years. With this approach, clinical differences were maintained only when there was a difference in costs, i.e. during treatment with IDegLira or iGlarLixi.

\section{Resource Use and Cost Data}

Costs were estimated from the perspective of the healthcare payer in the Czech Republic and expressed in 2018 CZK. Direct costs were included in the current analysis, capturing pharmacy costs, costs of diabetes-related complications and concomitant patient management costs.

Pharmacy costs were calculated based on resource use data obtained from DUAL II data for IDegLira (45.0 dose-steps per day) [13]. The mean difference in end-of-trial daily insulin doses between IDegLira and iGlarLixi reported by the ITC was applied to the IDegLira dose to obtain the daily iGlarLixi dose (48.6 dose-steps per day) [32]. Daily basal and bolus insulin doses were assumed to be equal following intensification after 5 years of fixed-ratio combination treatment in both arms, and were based on the DUAL VII study [14]. Throughout the analysis, patients were assumed to receive a daily concomitant metformin dose of $2000 \mathrm{mg}$. During treatment with IDegLira or iGlarLixi, patients were assumed to require one needle 
Table 2 Treatment effects applied in the analysis

\begin{tabular}{|c|c|c|c|}
\hline Parameter & $\begin{array}{l}\text { (1) Value applied in the } \\
\text { IDegLira arm (from } \\
\text { DUAL II [13]) }\end{array}$ & $\begin{array}{l}\text { (2) Between-treatment } \\
\text { difference: IDegLira versus } \\
\text { iGlarLixi (from ITC [32]) }\end{array}$ & $\begin{array}{l}\text { (3) Value applied in the } \\
\text { iGlarLixi arm [calculated } \\
\text { from (1) and (2)] }\end{array}$ \\
\hline HbAlc (\%) & $-1.92(0.07)$ & $\begin{array}{l}\text { Mean difference: }-0.44(-0.71 \\
\quad \text { to }-0.17)\end{array}$ & $-1.48(0.07)$ \\
\hline BMI $\left(\mathrm{kg} / \mathrm{m}^{2}\right)$ & $-0.92(0.43)$ & $\begin{array}{l}\text { Mean difference in body weight } \\
(\mathrm{kg})^{\mathrm{a}}:-1.42(-2.50 \text { to }- \\
0.35)\end{array}$ & $-0.41(0.43)$ \\
\hline $\begin{array}{l}\text { Severe hypoglycemic } \\
\text { events (per } 100 \\
\text { patient-years) }\end{array}$ & 1.10 & Rate ratio: $0.51(0.29-0.90)$ & 2.16 \\
\hline $\begin{array}{l}\text { Non-severe } \\
\text { hypoglycemic events } \\
\text { (per } 100 \text { patient- } \\
\text { years) }\end{array}$ & 152.3 & & 298.6 \\
\hline Daily dose (dose-steps) & 45.0 & $\begin{array}{l}\text { Mean difference: }-3.60(-10.3 \\
\text { to } 3.30)\end{array}$ & 48.6 \\
\hline
\end{tabular}

Values are presented as the mean with the SD in parenthesis for (1) and (3), and as the mean with the $95 \%$ confidence interval in parenthesis for (2)

ITC Indirect treatment comparison

${ }^{a}$ Differences in body weight were converted to differences in BMI based on the mean height $(168 \mathrm{~cm})$ of patients receiving IDegLira in DUAL II

and to perform one self-monitoring of blood glucose (SMBG) test, including use of one SMBG lancet and one test strip, per day. The number of needles and SMBG tests used was assumed to increase to four per day following treatment intensification.

Unit costs for medications, needles and SMBG testing were based on publicly available prices published by the Czech State Institute for Drug Control [35]. Reimbursement restrictions were accounted for in calculating the total annual treatment costs. Costs of complications were also obtained from published State Institute for Drug Control data (see Electronic Supplementary Material [ESM] Table S1 for cost values and detailed references).

\section{Health-State Utility Data}

Diabetes-related complications have been shown to be associated with reductions in health-related quality of life, and these reductions were captured in the analyses. Utilities were sourced from the published literature, including a systematic review of utilities for economic modeling in T2DM and a survey of quality-of-life loss associated with hypoglycemic events (see ESM Table S2) [36-39].

\section{Sensitivity Analyses}

Long-term projections of clinical and cost outcomes, based on short-term data, are associated with uncertainty [40]. Health economic guidance for the Czech Republic recommends addressing this uncertainty through the use of deterministic, one-way sensitivity analyses as well as probabilistic sensitivity analysis (PSA) [21].

In line with this guidance, a range of sensitivity analyses was conducted. The impact of the choice of time horizon was explored by 
using shorter time horizons (10 and 20 years). Importantly, as not all modeled patients died over these shorter time horizons, not all complications and costs were captured. The effect of discount rates on projected outcomes was investigated using lower (0\%) and higher (5\%) discount rates, as suggested by Czech guidance [21]. The influence of treatment effects was explored in several sensitivity analyses, including analyses in which the only between-treatment difference was assumed to be in HbA1c (with all other treatment effects, hypoglycemia rates and daily fixed-ratio combination doses equal to those in the iGlarLixi arm), in which HbA1c progression was assumed to follow the UK Prospective Diabetes Study (UKPDS) HbA1c progression built into the CDM, and in which the upper and lower 95\% confidence interval bounds reported by the ITC for the estimated between-treatment difference in $\mathrm{HbA1c}$ were used (Table 2) [24, 32]. Similarly, the influence of BMI was assessed by running analyses in which the BMI difference between treatments was abolished (with all other treatment effects, hypoglycemia rates and daily doses applied as in the base case), in which the BMI difference between treatments was maintained over patient lifetimes, and in which the upper and lower 95\% confidence interval bounds reported by the ITC for estimated between-treatment difference in BMI were used (TableI2) [32]. In addition, sensitivity analyses were conducted in which the difference in hypoglycemic event rates between treatments was set to zero (with all other treatment effects, hypoglycemia rates and daily doses applied as in the base case), and in which only statistically significant differences in HbA1c, BMI and severe hypoglycemic events (SHE) rates were applied while daily insulin doses and non-severe hypoglycemic event rates were set to those of the iGlarLixi arm in both arms.

Alternative treatment switching patterns were also explored by assuming earlier (after 3 years) and later (7 years) switches to basalbolus therapy than in the base case ( 5 years). The impact of the choice of risk equation was investigated by conducting a sensitivity analysis using the UKPDS 82 equation built into the CDM. Use of this risk equation is recommended by the CDM proprietors for sensitivity analysis [26].

The effect of over- or under-estimation of direct costs of diabetes-related complication was explored in two sensitivity analyses. In the first, the cost of treating complications was increased by $10 \%$; in the second, the cost was decreased by $10 \%$. Disutilities were also varied for hypoglycemic events and BMI [41, 42]. In addition, a sensitivity analysis was conducted assuming diminishing disutilities for non-severe hypoglycemic events [43].

The PSA was performed by using the functionality provided by the CDM, which samples complication costs, treatment effects and cohort characteristics from distributions and feeds the sampled values to second-order Monte Carlo simulations.

\section{Statement of Ethics Compliance}

This article is based on previously conducted studies and does not contain any studies with human participants or animals performed by any of the authors.

\section{RESULTS}

\section{Base Case Analysis}

Long-term projections suggested that in comparison to iGlarLixi, IDegLira was associated with clinical benefits (Table 3). Compared to iGlarLixi, IDegLira was associated with gains in discounted life expectancy of 0.11 years and in discounted QALE of 0.14 QALYs. The clinical benefits of treatment with IDegLira relative to iGlarLixi resulted from a reduced incidence and delayed onset of diabetes-related complications (Fig. 1).

The clinical benefits associated with IDegLira came at an increased cost. Relative to the iGlarLixi pen containing $33 \mu \mathrm{g} / \mathrm{mL}$ of lixisenatide, lifetime direct medical costs for patients treated with IDegLira were approximately CZK 94,029 higher per patient (Table 3). The corresponding incremental cost relative to the iGlarLixi pen containing $50 \mu \mathrm{g} / \mathrm{mL}$ of 
Table 3 Long-term cost-effectiveness outcomes

\begin{tabular}{llll}
\hline Health outcomes & IDegLira & iGlarLixi $\mathbf{3 3} \mathbf{\mu g}^{\mathbf{a}}$ & Difference \\
\hline Discounted life expectancy (years) & 13.92 & 13.81 & +0.11 \\
$\begin{array}{l}\text { Discounted quality-adjusted life } \\
\text { expectancy (QALYs) }\end{array}$ & 8.98 & 8.84 & +0.14 \\
Discounted direct costs (CZK) & & & \\
ICER & 878,839 & 784,810 & $+94,029$ \\
\hline Health outcomes & CZK 695,998 per QALY gained & \\
\hline Discounted life expectancy (years) & IDegLira & iGlarLixi $\mathbf{5 0} \mathbf{\mu g}^{\mathbf{a}}$ & Difference \\
Discounted quality-adjusted life & 13.92 & 13.81 & +0.11 \\
$\quad$ expectancy (QALYs) & 8.98 & 8.84 & +0.14 \\
Discounted direct costs (CZK) & & & \\
ICER & 878,839 & 831,781 & $+47,058$ \\
\hline
\end{tabular}

$C Z K$ Czech koruna, ICER incremental cost-effectiveness ratio, $Q A L Y$ quality-adjusted life-years

${ }^{a}$ iGlarLixi $33 \mu \mathrm{g}$ denotes the iGlarLixi pen containing $33 \mu \mathrm{g} / \mathrm{mL}$ lixisenatide, while iGlarLixi $50 \mu \mathrm{g}$ denotes the iGlarLixi pen containing $50 \mu \mathrm{g} / \mathrm{mL}$ lixisenatide

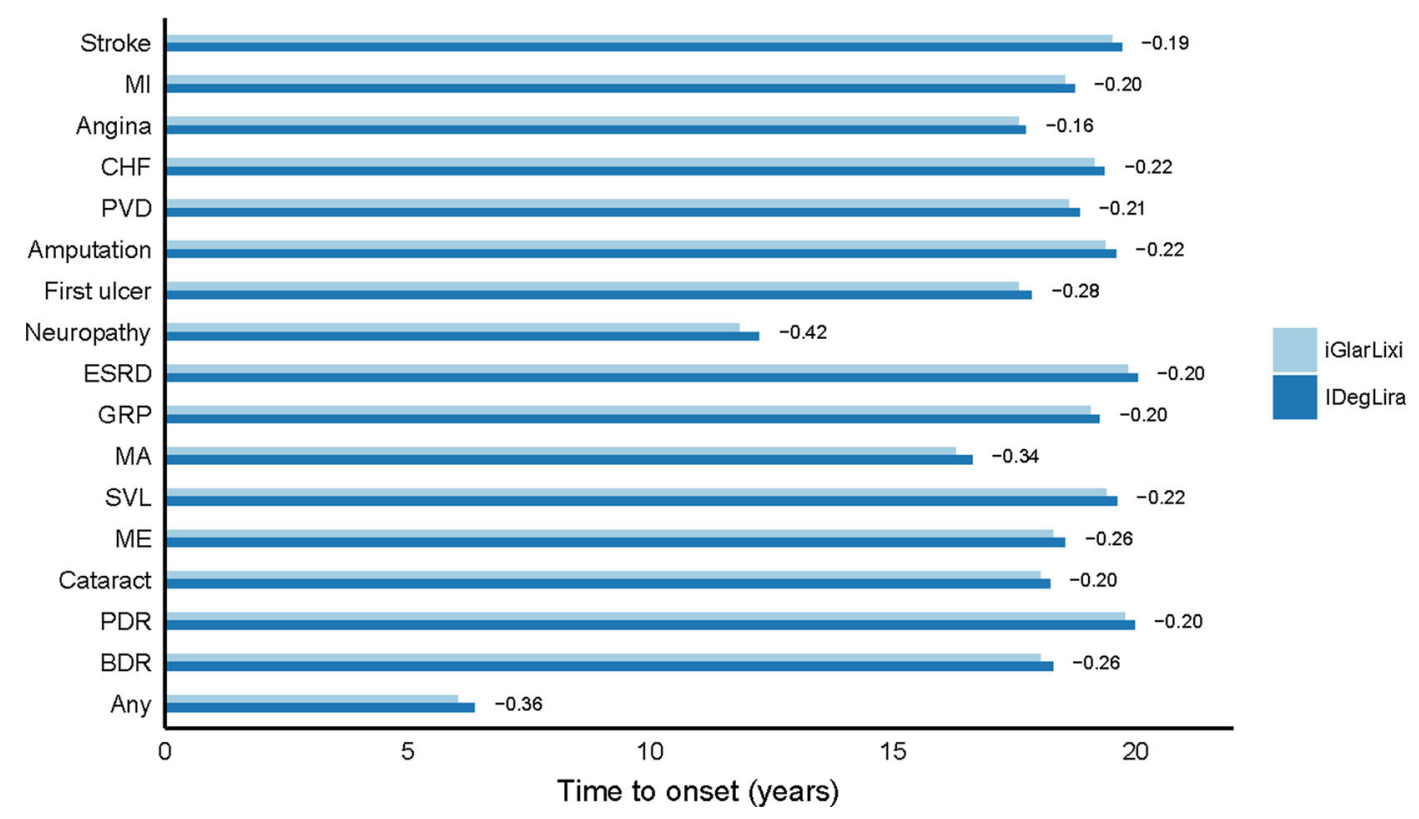

Fig. 1 Mean time to complication onset. $B D R$ Background diabetic retinopathy, $C H F$ congestive heart failure, $E S R D$ end-stage renal disease, GRP gross proteinuria, $M A$

lixisenatide was CZK 47,058. The higher incremental costs for IDegLira were due to higher IDegLira acquisition costs over the first 5 years microalbuminuria, $M E$ macular edema, $M I$ myocardial infarction, $P D R$ proliferative diabetic retinopathy, $P V D$ peripheral vascular disease, $S V L$ severe vision loss

of the analysis, which, however, were partially offset by reduced costs for the treatment of diabetes-related complications. In particular, 
costs associated with renal- and neuropathy-related complications were reduced by CZK 3893 and CZK 3686, respectively, in patients treated with IDegLira relative to those on iGlarLixi.

Estimation of the long-term clinical outcomes indicated that both life expectancy and QALE were improved with IDegLira treatment compared with iGlarLixi treatment, at an increased cost from a healthcare payer perspective. IDegLira was associated with an ICER of CZK 695,998 per QALY gained versus the iGlarLixi pen containing $33 \mu \mathrm{g} / \mathrm{mL}$ of lixisenatide and of CZK 348,323 per QALY gained versus the iGlarLixi pen containing $50 \mu \mathrm{g} / \mathrm{mL}$ of lixisenatide (Table 3). As these ICERs fell below the commonly used WTP threshold, IDegLira is likely to be considered cost-effective versus iGlarLixi.

\section{Deterministic Sensitivity Analyses}

Deterministic sensitivity analyses demonstrated that the projected cost-effectiveness of IDegLira relative to iGlarLixi was generally not sensitive to changes in input data or assumptions (Fig. 2; ESM Tables S3 and S4).
Shorter simulated time horizons were associated with increases in ICER relative to the base case. When a shorter time horizon was used, the incremental clinical benefit of IDegLira was smaller as long-term benefits were not captured in full, indicating that a lifetime perspective is indeed appropriate, as recommended by health economic guidance. Still, most ICERs calculated for shortened time horizons fell below the WTP threshold, suggesting that IDegLira was costeffective versus iGlarLixi even when some of the clinical benefits of IDegLira were not accounted for. When no discounting was applied, the ICER decreased, while the opposite effect was observed for a discount rate of 5\% per annum.

A difference in cost-effectiveness outcomes was observed when treatments were assumed to differ in HbA1c only, i.e. if the benefits of IDegLira on BMI, hypoglycemia rate and daily doses were not accounted for. In this analysis, ICERs increased relative to the base case, suggesting that the non-HbA1c benefits of IDegLira are also important drivers of clinical and cost outcomes.

Abolishing the difference in BMI between treatments increased the ICER while

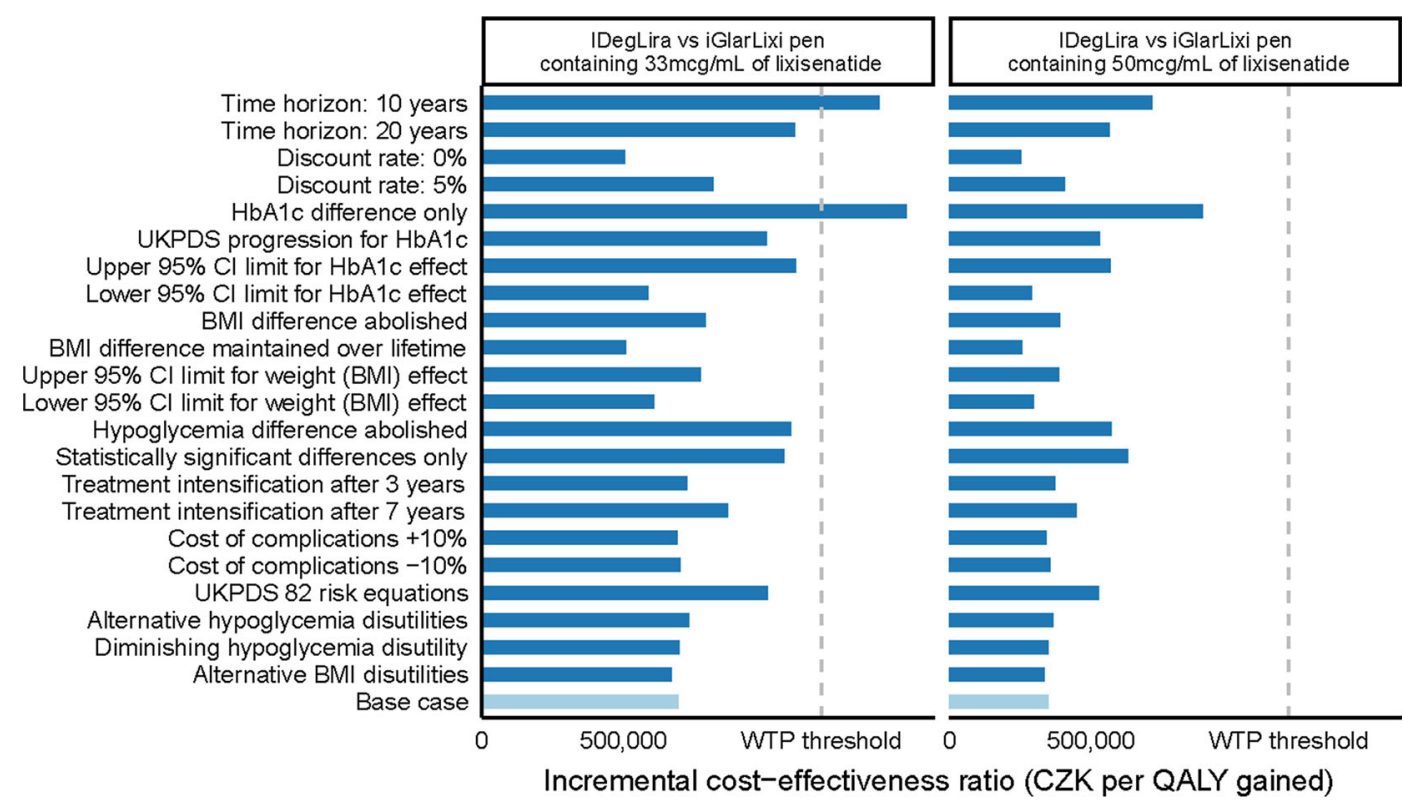

Fig. 2 Results of deterministic sensitivity analyses. $B M I$ Body mass index, $C I$ confidence interval, $C Z K$ Czech koruna, $H b A 1 c$ glycated hemoglobin, $Q A L Y$ quality- adjusted life-year, UKPDS United Kingdom Prospective Diabetes Study, WTP willingness to pay 
maintaining the difference over patient lifetimes decreased the ICER. Using the lower 95\% confidence interval bound for the treatment effect on weight reduced the clinical benefit associated with IDegLira over iGlarLixi, thereby increasing the ICER relative to the base case as incremental costs remained unchanged (although the ICER still fell below the WTP threshold). Conversely, using the upper bound of the $95 \%$ confidence interval for the treatment effect on weight increased the clinical benefit associated with IDegLira and, given unchanged costs, reduced the ICER relative to the base case.

Similarly, when the difference in hypoglycemic event rates was abolished, the ICER increased relative to the base case due to the reduced incremental clinical benefit of IDegLira and slight increase in incremental costs. The same pattern was observed when only statistically significant differences in treatment effects were considered. Assumptions regarding the timing of intensification to basal-bolus insulin also did not affect conclusions regarding costeffectiveness. Treatment switching after 3 years led to a small increase in the ICER as the reduction in incremental clinical benefits associated with IDegLira was slightly larger than the reduction in incremental acquisition costs. When intensification occurred after 7 years of fixed-ratio combination treatment, a small increase in the ICER was also observed due to the fact that the increased clinical gains associated with IDegLira were offset by higher acquisition costs.

Higher complication costs were associated with a reduced ICER relative to the base case, while the converse was observed for lower complication costs. Use of the UKPDS 82 risk equations for the prediction of cardiovascular events was associated with an increase in ICERs, but IDegLira was still considered to be cost-effective versus both iGlarLixi pens. The use of an alternative disutility associated with BMI and hypoglycemia, as well as assuming a diminishing disutilities approach for hypoglycemic events, did not have a large impact on the ICER, indicating that cost-effectiveness outcomes were not driven by these assumptions.

\section{Probabilistic Sensitivity Analysis}

The PSA conducted with sampling around cohort characteristics, treatment effects, complication costs and utilities produced mean results similar to those for the base case. For the comparison of IDegLira with the iGlarLixi pen containing $33 \mu \mathrm{g} / \mathrm{mL}$ of lixisenatide, the mean improvement in QALE gained with IDegLira versus iGlarLixi was 0.12 QALYs, at mean incremental costs of CZK 99,703, giving an ICER of CZK 843,898 per QALY gained. The corresponding values for the comparison with the iGlarLixi pen containing $50 \mu \mathrm{g} / \mathrm{mL}$ of lixisenatide were 0.12 QALYs at incremental costs of CZK 50,716, yielding an ICER of CZK 440,739 per QALY gained.

Cost-effectiveness scatterplots, which were based on 1000 simulated cohorts of 1000 patients, showed that most of the sampled ICER fell in the upper right quadrant (74 and 66\% for the comparison of IDegLira with the iGlarLixi pen containing 33 and $50 \mu \mathrm{g} / \mathrm{mL}$ of lixisenatide, respectively), indicating that IDegLira was associated with increased effectiveness and costs (Fig. 3). At a WTP threshold of CZK 1,200,000 per QALY gained, there was a $59 \%$ probability that IDegLira was cost-effective versus the iGlarLixi pen containing $33 \mu \mathrm{g} / \mathrm{mL}$ of lixisenatide, and a $68 \%$ probability that IDegLira was cost-effective versus the iGlarLixi pen containing $50 \mu \mathrm{g} / \mathrm{mL}$ of lixisenatide.

\section{DISCUSSION}

The present analysis assessed the cost-effectiveness of IDegLira versus iGlarLixi in the Czech Republic for patients with T2DM who were inadequately controlled on basal insulin. Based on clinical data from the DUAL II trial and an ITC comparing IDegLira with iGlarLixi, outcomes were projected over patient lifetimes using a validated health economic model $[13,24,32]$. At a WTP threshold of CZK $1,200,000$ per QALY gained, IDegLira was found to be cost-effective versus both iGlarLixi pens currently available (containing 33 and $50 \mu \mathrm{g} /$ $\mathrm{mL}$ of lixisenatide, respectively). Improvements in HbA1c and BMI in patients treated with 


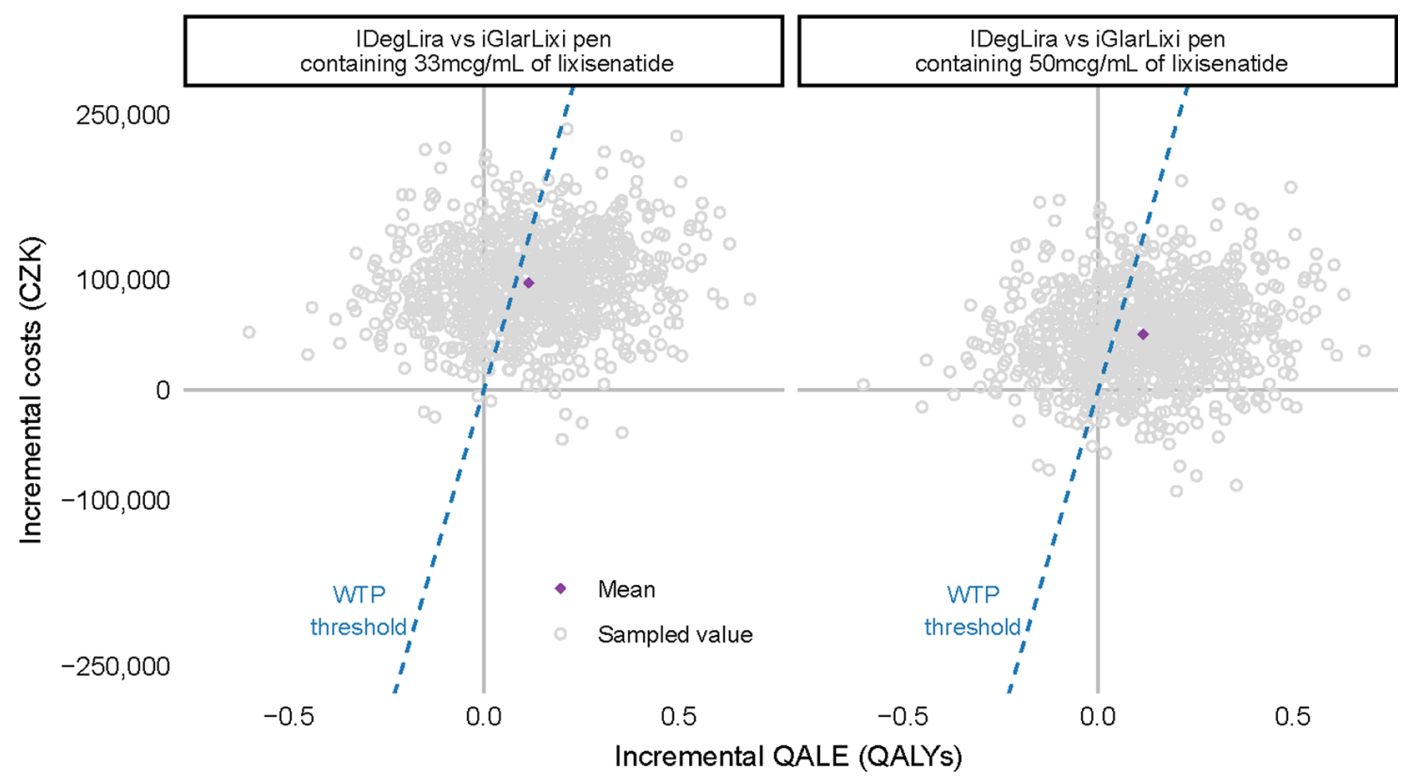

Fig. 3 Cost-effectiveness scatterplot. QALE Quality-adjusted life expectancy, WTP willingness to pay, QALY Qualityadjusted life-year, $C Z K$ Czech koruna

IDegLira led to reductions in cumulative incidence and delayed onset of diabetes-related complications, resulting in increased life expectancy and QALE. IDegLira was associated with higher treatment costs than iGlarLixi, a reflection of the former's higher acquisition costs; however, these were partially offset by lower costs of treating diabetes-related complications, due to complications avoided or delayed in patients treated with IDegLira. Over patient lifetimes, IDegLira was associated with a gain of 0.14 QALYs and incremental costs of CZK 94,029 and CZK 47,058 versus iGlarLixi pens containing 33 and $50 \mu \mathrm{g} / \mathrm{mL}$ of lixisenatide, respectively, leading to ICER of CZK 695,998 and CZK 348,323 per QALY gained. The sensitivity analyses suggested that the estimated treatment difference for $\mathrm{HbA} 1 \mathrm{c}$ as well as differences in hypoglycemia rates were key drivers of the results. In addition, the sensitivity analyses demonstrated that IDegLira was associated with benefits to patients over their entire lifetime, so long-term analyses were required to fully capture the impact of IDegLira.

While no previous health economic evaluation of IDegLira versus iGlarLixi is available, the results of the present analysis analysis are broadly aligned with those obtained in earlier studies that showed IDegLira to be cost-effective versus its competitors in a range of settings. In a recent CEA conducted for the Czech setting, IDegLira was compared with insulin intensification regimens in patients with T2DM inadequately controlled on basal insulin [20]. The authors of this study considered IDegLira to be cost-effective versus both basal-bolus therapy and basal insulin plus GLP-1 receptor agonist therapy from the perspective of the healthcare payer in the Czech Republic. Similar long-term analyses have been conducted in the UK, the Netherlands, Sweden and Slovakia, all of which showed IDegLira to be cost-effective versus basal-bolus insulin and/or basal insulin plus GLP-1 receptor agonists from the perspective of the respective healthcare payer [44-47]. For the UK, a recent short-term CEA also showed that IDegLira was likely to be cost-effective even over 1 year relative to treatment with basal-bolus insulin therapy [48].

To date, no head-to-head clinical trials comparing IDegLira with iGlarLixi have been conducted. Therefore, data from a previously published ITC were used to compare the two fixed-ratio combinations, which may be considered a limitation of the present analysis [37]. Evidence synthesis methods, such as indirect 
comparison or meta-analysis, have become widely used and accepted in health technology assessments and health economic modeling, particularly to provide healthcare payers and decision-makers with robust evidence even if no head-to-head clinical data are available $[49,50]$. In addition, the clinical data used in the present analysis were sourced from a consistent source, namely the DUAL clinical trial program investigating IDegLira. An additional limitation of the analysis was the reliance on relatively shortterm clinical trial data (pooled in the ITC) as the base for long-term projections of clinical and economic outcomes, which were therefore associated with uncertainty. Despite this limitation, which is common to many health economic analyses, projections of outcomes over patient lifetimes are recommended in guidelines for the economic evaluation of interventions for patients with diabetes as a means to inform decision-making even if long-term clinical data are not available $[5,27]$. In the present analysis, every effort was made to reduce the uncertainty surrounding the long-term outcomes by using a diabetes model which has been extensively published and validated against real-life data [24-26]. In addition, extensive sensitivity analyses were conducted, and these consistently showed IDegLira to be cost-effective relative to iGlarLixi.

Clinical trials have consistently demonstrated improved short-term clinical outcomes associated with IDegLira in patients with T2DM who failed to achieve glycemic control on basal insulin alone $[13,14]$. The present analysis indicated that shortterm outcomes translated into long-term benefits. Importantly, outcomes from clinical trials have been confirmed by real-world studies. In a chart review of more than 600 European patients with T2DM who initiated IDegLira at least 6 months before the review was performed, IDegLira was associated with a $0.9 \%$ reduction in HbA1c at 6 months after treatment initiation relative to baseline [51]. Similarly, reductions have been observed for weight $(0.7 \mathrm{~kg}$ at 6 months vs. baseline) and for use of concomitant antidiabetic medications. These findings were confirmed by an observational study of patients with T2DM in Switzerland, in whom reductions in $\mathrm{HbA1c}$ and BMI were observed over a 6-month period following initiation of IDegLira therapy; in addition, required insulin doses were reduced, without SHE over the course of the 6 months [52]. The benefits associated with IDegLira are reflected in physicians' perceptions, as reported by a survey of 235 primary and secondary care physicians from Europe [53]. Based on their experience in clinical practice, physicians reported being more satisfied with prescribing IDegLira than basal-bolus insulin to achieve important treatment targets, including avoidance of weight gain and hypoglycemia, while also considering IDegLira to be simpler to administer [53]. Importantly, the simplicity of IDegLira therapy, which is matched by reports of higher patient satisfaction and easier training of patients, has been suggested as an important component of approaches to reduce treatment intensification inertia [54].

\section{CONCLUSION}

From the perspective of the Czech healthcare payer, IDegLira is likely to be cost-effective versus iGlarLixi in the treatment of patients with T2DM who are uncontrolled on basal insulin. IDegLira was associated with a low risk of hypoglycemia and reduced cumulative incidence as well as delayed onset of diabetes-related complications, which improved quality of life and reduced the costs of treating diabetesrelated complications. IDegLira is therefore a valuable option for intensification of antidiabetic therapy in the Czech Republic.

\section{ACKNOWLEDGEMENTS}

Funding. This study and any processing charges related to the submission of the manuscript were funded by Novo Nordisk. All authors had full access to all of the data in this study and take complete responsibility for the integrity of the data and accuracy of the data analysis.

Authorship. All named authors meet the International Committee of Medical Journal Editors (ICMJE) criteria for authorship for this 
article, take responsibility for the integrity of the work as a whole, and have given their approval for this version to be published.

Disclosures. Johannes Pöhlmann is an employee of Ossian Health Economics and Communications, which received consulting fees from Novo Nordisk for conducting the analysis and preparing the present article. Monika Russel-Szymczyk is an employee of Novo Nordisk Pharma Sp. z.o.o. Pavel Holik is an employee of Novo Nordisk s.r.o. Karel Rychna is an employee of Novo Nordisk s.r.o. Barnaby Hunt is an employee of Ossian Health Economics and Communications, which received consulting fees from Novo Nordisk for conducting the analysis and preparing the present article.

Compliance with Ethics Guidelines. This article is based on previously conducted studies and does not contain any studies with human participants or animals performed by any of the authors.

Data Availability. The datasets used and/or analyzed during the current study, in addition to those made available in the Electronic Supplementary Material, are available from the corresponding author on reasonable request.

Open Access. This article is distributed under the terms of the Creative Commons Attribution-NonCommercial 4.0 International License (http://creativecommons.org/licenses/ by-nc/4.0/), which permits any noncommercial use, distribution, and reproduction in any medium, provided you give appropriate credit to the original author(s) and the source, provide a link to the Creative Commons license, and indicate if changes were made.

\section{REFERENCES}

1. $\mathrm{Hu}$ FB, Satija A, Manson JE. Curbing the diabetes pandemic: the need for global policy solutions. JAMA. 2015;313(23):2319-20. https://doi.org/10. 1001/jama.2015.5287.
2. Ústavu zdravotnických informací a statistiky České republiky (Institute of Health Information and Statistics of the Czech Republic [UZIS]). Stručný přehled činnosti oboru diabetologie a endokrinologie za období 2007-2017 (Brief overview of diabetology and endocrinology in the period 2007-2017). 2018. http://www.uzis.cz/system/files/ nzis_rep_2018_K01_A004_diabet_endokrin_2017. pdf. Accessed 10 Dec 2018.

3. United Kingdom Prospective Diabetes Study Group. Effect of intensive blood-glucose control with metformin on complications in overweight patients with type 2 diabetes (UKPDS 34)). Lancet. 1998;352(9131):854-65. https://doi.org/10.1016/ s0140-6736(98)07037-8.

4. Morales J, Merker L. Minimizing hypoglycemia and weight gain with intensive glucose control: potential benefits of a new combination therapy (IDegLira). Adv Ther. 2015;32(5):391-403. https://doi. org/10.1007/s12325-015-0208-2.

5. Česká diabetologická společnost (Czech Diabetes Society). Doporučený postup péče o diabetes mellitus 2. typu $-2017+$ pŕíloha Algoritmus terapie (Treatment guidelines for type 2 diabetes2017 + treatment algorithm]) 2017. http://www. diab.cz/dokumenty/standard_lecba_dm_typ_II.pdf and http://www.diab.cz/dokumenty/priloha algoritmus_terapie.pdf. Accessed 10 Dec 2018.

6. Brož J, Janíčková Žd'árská D, Urbanová J, et al. Current level of glycemic control and clinical inertia in subjects using insulin for the treatment of type 1 and type 2 diabetes in the Czech Republic and the Slovak Republic: results of a multinational, multicenter, observational survey (DIAINFORM). Diabetes Ther. 2018;9(5):1897-906. https://doi.org/ 10.1007/s13300-018-0485-2.

7. Inman TR, Plyushko E, Austin NP, Johnson JL. The role of basal insulin and GLP-1 receptor agonist combination products in the management of type 2 diabetes. Ther Adv Endocrinol Metab. 2018; 9(5):151-5. https://doi.org/10.1177/204201881876 3698.

8. Maiorino MI, Chiodini P, Bellastella G, Capuano A, Esposito K, Giugliano D. Insulin and glucagon-like peptide 1 receptor agonist combination therapy in type 2 diabetes: a systematic review and metaanalysis of randomized controlled trials. Diabetes Care. 2017;40(4):614-24. https://doi.org/10.2337/ dc16-1957.

9. Valentine V, Goldman J, Shubrook JH. Rationale for, initiation and titration of the basal insulin/ GLP-1RA fixed-ratio combination products, IDegLira and iGlarLixi, for the management of type 2 diabetes. Diabetes Ther. 2017;8(4):739-52. https:// doi.org/10.1007/s13300-017-0287-y. 
10. Gough SC, Bode B, Woo V, et al. Efficacy and safety of a fixed-ratio combination of insulin degludec and liraglutide (IDegLira) compared with its components given alone: results of a phase 3, open-label, randomised, 26-week, treat-to-target trial in insulin-naive patients with type 2 diabetes. Lancet Diabetes Endocrinol. 2014;2(11):885-93. https:// doi.org/10.1016/S2213-8587(14)70174-3.

11. Linjawi S, Bode BW, Chaykin LB, et al. The efficacy of IDegLira (insulin degludec/liraglutide combination) in adults with type 2 diabetes inadequately controlled with a GLP-1 receptor agonist and oral therapy: DUAL III randomized clinical trial. Diabetes Ther. 2017;8(1):101-14. https://doi.org/10. 1007/s13300-016-0218-3.

12. Rodbard HW, Bode BW, Harris SB, et al. Safety and efficacy of insulin degludec/liraglutide (IDegLira) added to sulphonylurea alone or to sulphonylurea and metformin in insulin-naïve people with Type 2 diabetes: the DUAL IV trial. Diabet Med. 2017;34(2):189-96. https://doi.org/10.1111/dme. 13256 .

13. Buse JB, Vilsbøll T, Thurman J, et al. Contribution of liraglutide in the fixed-ratio combination of insulin degludec and liraglutide (IDegLira). Diabetes Care. 2014;37(11):2926-33. https://doi.org/ 10.2337/dc14-0785.

14. Billings LK, Doshi A, Gouet D, et al. Efficacy and safety of IDegLira versus basal-bolus insulin therapy in patients with type 2 diabetes uncontrolled on metformin and basal insulin: the DUAL VII randomized clinical trial. Diabetes Care. 2018;41(5):1009-16. https://doi.org/10.2337/dc171114.

15. King AB, Philis-Tsimikas A, Kilpatrick ES, Langbakke $\mathrm{IH}$, Begtrup K, Vilsbøll T. A fixed ratio combination of insulin degludec and liraglutide (IDegLira) reduces glycemic fluctuation and brings more patients with type 2 diabetes within blood glucose target ranges. Diabetes Technol Ther. 2017;19(4):255-64. https://doi.org/10.1089/dia. 2016.0405 .

16. Aroda VR, Rosenstock J, Wysham C, et al. Efficacy and safety of LixiLan, a titratable fixed-ratio combination of insulin glargine plus lixisenatide in type 2 diabetes inadequately controlled on basal insulin and metformin: the LixiLan-L randomized trial. Diabetes Care. 2016;39(11):1972-80. https://doi. org/10.2337/dc16-1495.

17. Rosenstock J, Aronson R, Grunberger G, et al. Benefits of LixiLan, a titratable fixed-ratio combination of insulin glargine plus lixisenatide, versus insulin glargine and lixisenatide monocomponents in type 2 diabetes inadequately controlled on oral agents: the LixiLan-O randomized trial. Diabetes Care.
2016;39(11):2026-35. dc16-0917.

https://doi.org/10.2337/

18. Weinstein MC, Stason WB. Foundations of cost-effectiveness analysis for health and medical practices. N Engl J Med. 1977;296(13):716-21. https:// doi.org/10.1056/NEJM197703312961304.

19. Detsky AS, Naglie IG. A clinician's guide to costeffectiveness analysis. Ann Intern Med. 1990;113(2):147-54. https://doi.org/10.7326/00034819-113-2-147.

20. Kvapil M, Prázný M, Holik P, Rychna K, Hunt B. Cost-effectiveness of IDegLira versus insulin intensification regimens for the treatment of adults with type 2 diabetes in the Czech Republic. Diabetes Ther. 2017;8(6):1331-47. https://doi.org/10.1007/ s13300-017-0323-y.

21. Státní stav pro kontrolu léčiv (State Institute for Drug Control). Postup pro posuzování analýzy nákladové efektivity (Guidelines for cost-effectiveness analysis). 2017. https://www.sukl.cz/file/ 85788_1_1. Accessed 10 Dec 2018.

22. International Society for Pharmacoeconomics and Outcomes Research (ISPOR). Pharmacoeconomic guidelines around the world-country/region: Czech Republic. 2018. https://tools.ispor.org/ PEguidelines/countrydet.asp?c=47\&t=2. Accessed 10 Dec 2018.

23. Marseille E, Larson B, Kazi DS, Kahn JG, Rosen S. Thresholds for the cost-effectiveness of interventions: alternative approaches. Bull World Health Organ. 2015;93(2):118-24. https://doi.org/10.2471/ BLT.14.138206.

24. Palmer AJ, Roze S, Valentine WJ, et al. The CORE Diabetes Model: projecting long-term clinical outcomes, costs and cost-effectiveness of interventions in diabetes mellitus (types 1 and 2) to support clinical and reimbursement decision-making. Curr Med Res Opin. 2004;20[Suppl 1]:S5-26. https://doi. org/10.1185/030079904X1980.

25. Palmer AJ, Roze S, Valentine WJ, et al. Validation of the CORE Diabetes Model against epidemiological and clinical studies. Curr Med Res Opin. 2004;20[Suppl 1]:S27-40. https://doi.org/10.1185/ 030079904 X2006.

26. McEwan P, Foos V, Palmer JL, Lamotte M, Lloyd A, Grant D. Validation of the IMS CORE diabetes model. Value Health. 2014;17(6):714-24. https:// doi.org/10.1016/j.jval.2014.07.007.

27. American Diabetes Association Consensus Panel. Guidelines for computer modeling of diabetes and its complications: guidelines for computer modeling of diabetes and its complications. Diabetes 
Care. 2004;27(9):2262-25. https://doi.org/10.2337/ diacare.27.9.2262.

28. World Health Organization. Global Health Observatory (GHO) data: life tables by country: Czechia. 2017. http://apps.who.int/gho/data/view.main. 60430?lang=en. Accessed 10 Nov 2018.

29. Attema AE, Brouwer WBF, Claxton K. Discounting in economic evaluations. Pharmacoeconomics. 2018;36(7):745-58. https://doi.org/10.1007/s402 73-018-0672-z.

30. American Cancer Society, Inc. and Vital Strategies. The tobacco atlas. Czech Republic. 2018. https:// tobaccoatlas.org/country/czech-republic/. Accessed 10 Dec 2018.

31. World Health Organization. Substance abuse: Czech Republic. 2014. http://www.who.int/sub stance_abuse/publications/global_alcohol_report/ profiles/cze.pdf. Accessed 10 Dec 2018.

32. Evans M, Billings LK, Håkan-Bloch J, et al. An indirect treatment comparison of the efficacy of insulin degludec/liraglutide (IDegLira) and insulin glargine/lixisenatide (iGlarLixi) in patients with type 2 diabetes uncontrolled on basal insulin. J Med Econ. 2018;21(4):340-7. https://doi.org/10.1080/ 13696998.2017.1409228.

33. Jansen JP, Fleurence R, Devine B, et al. Interpreting indirect treatment comparisons and network metaanalysis for health-care decision making: report of the ISPOR Task Force on Indirect Treatment Comparisons Good Research Practices: part 1. Value Health. 2011;14(4):417-28. https://doi.org/10. 1016/j.jval.2011.04.002.

34. Hoaglin DC, Hawkins N, Jansen JP, et al. Conducting indirect-treatment-comparison and network-meta-analysis studies: report of the ISPOR Task Force on Indirect Treatment Comparisons Good Research Practices: part 2. Value Health. 2011;14(4):429-37. https://doi.org/10.1016/j.jval. 2011.01.011.

35. Státní stav pro kontrolu léčiv (State Institute for Drug Control): Medicinal Products Database. 2018. http://www.sukl.eu/modules/medication/search. php. Accessed 10 Dec 2018.

36. Beaudet A, Clegg J, Thuresson PO, Lloyd A, McEwan P. Review of utility values for economic modeling in type 2 diabetes. Value Health. 2014;17(4):462-70. https://doi.org/10.1016/j.jval. 2014.03.003.

37. Evans M, Khunti K, Mamdani M, et al. Health-related quality of life associated with daytime and nocturnal hypoglycaemic events: a time trade-off survey in five countries. Health Qual Life
Outcomes. 2013;11:90. https://doi.org/10.1186/ 1477-7525-11-90.

38. Bagust A, Beale S. Modelling EuroQol health-related utility values for diabetic complications from CODE-2 data. Health Econ. 2005;14(3):217-30. https://doi.org/10.1002/hec.910.

39. Wasserfallen JB, Halabi G, Saudan P, et al. Quality of life on chronic dialysis: comparison between haemodialysis and peritoneal dialysis. Nephrol Dial Transplant. 2004;19(6):1594-9. https://doi.org/10. 1093/ndt/gfh175.

40. Briggs AH, Weinstein MC, Fenwick EA, et al. Model parameter estimation and uncertainty: a report of the ISPOR-SMDM modeling good research practices task force-6. Value Health. 2012;15(6):835-42. https://doi.org/10.1016/j.jval.2012.04.014.

41. Currie CJ, Morgan CL, Poole CD, Sharplin P, Lammert M, McEwan P. Multivariate models of healthrelated utility and the fear of hypoglycaemia in people with diabetes. Curr Med Res Opin. 2006; 22(8):1523-34. https://doi.org/10.1185/03007990 $6 \times 115757$.

42. Lee AJ, Morgan CL, Morrissey M, Wittrup-Jensen $\mathrm{KU}$, Kennedy-Martin T, Currie CJ. Evaluation of the association between the EQ-5D (health-related utility) and body mass index (obesity) in hospitaltreated people with type 1 diabetes, type 2 diabetes and with no diagnosed diabetes. Diabet Med. 2005;22(11):1482-6. https://doi.org/10.1111/j. 1464-5491.2005.01657.x.

43. Lauridsen JT, Lønborg J, Gundgaard J, Jensen HH. Diminishing marginal disutility of hypoglycaemic events: results from a time trade-off survey in five countries. Qual Life Res. 2014;23(9):2645-50. https://doi.org/10.1007/s11136-014-0712-x.

44. Davies MJ, Glah D, Chubb B, Konidaris G, McEwan P. Cost effectiveness of IDegLira vs. alternative basal insulin intensification therapies in patients with type 2 diabetes mellitus uncontrolled on basal insulin in a UK setting. Pharmacoeconomics. 2016;34(9):953-66. https://doi.org/10.1007/s402 73-016-0433-9.

45. Hunt B, Glah D, van der Vliet M. Modeling the long-term cost-effectiveness of IDegLira in patients with type 2 diabetes who are failing to meet glycemic targets on basal insulin alone in the Netherlands. Diabetes Ther. 2017;8(4):753-65. https://doi. org/10.1007/s13300-017-0266-3.

46. Ericsson $\AA$, Lundqvist A. Cost effectiveness of insulin degludec plus liraglutide (IDegLira) in a fixed combination for uncontrolled type 2 diabetes mellitus in Sweden. Appl Health Econ Health 
Policy. 2017;15(2):237-48. https://doi.org/10.1007/ s40258-016-0301-y.

47. Psota M, Psenkova MB, Racekova N, de Arellano RA, Vandebrouck T, Hunt B. Cost-effectiveness analysis of IDegLira versus basal-bolus insulin for patients with type 2 diabetes in the Slovak health system. Clin Outcomes Res. 2017;9:749-62. https://doi.org/ 10.2147/ceor.s143127.

48. Drummond R, Malkin S, Du Preez M, Lee XY, Hunt $\mathrm{B}$. The management of type 2 diabetes with fixedratio combination insulin degludec/liraglutide (IDegLira) versus basal-bolus therapy (insulin glargine U100 plus insulin aspart): a short-term costeffectiveness analysis in the UK setting. Diabetes Obes Metab. 2018;20(10):2371-8. https://doi.org/ 10.1111/dom.13375.

49. Dias S, Welton NJ, Sutton AJ, Ades AE. Introduction to evidence synthesis for decision making: NICE DSU Technical Support Document No. 1. 2012. National Institute for Health and Care Excellence (NICE): NICE Decision Support Unit Technical Support Documents. 2018. https://www.ncbi.nlm. nih.gov/pubmedhealth/PMH0089906/. Accessed 10 Dec 2018.

50. Shinkins B, Yang Y, Abel L, Fanshawe TR. Evidence synthesis to inform model-based cost-effectiveness evaluations of diagnostic tests: a methodological review of health technology assessments. BMC Med Res Methodol. 2017;17(1):56. https://doi.org/10. 1186/s12874-017-0331-7.

51. Price H, Blüher M, Prager R, Phan TM, Thorsted BL, Schultes B. Use and effectiveness of a fixed-ratio combination of insulin degludec/liraglutide (IDegLira) in a real-world population with type 2 diabetes: results from a European, multicentre, retrospective chart review study. Diabetes Obes Metab. 2018;20(4):954-62. https://doi.org/10.1111/ dom.13182.

52. Sofra D. Glycemic control in a real-life setting in patients with type 2 diabetes treated with IDegLira at a single Swiss Center. Diabetes Ther. 2017;8(2):377-84. https://doi.org/10.1007/s13300017-0234-y.

53. Drummond R, Baru A, Dutkiewicz M, Basse A, Tengmark BO. Physicians' real-world experience with IDegLira: results of a European survey. BMJ Open Diabetes Res Care. 2018;6(1):e000531. https://doi.org/10.1136/bmjdrc-2018-000531.

54. Russell-Jones D, Pouwer F, Khunti K. Identification of barriers to insulin therapy and approaches to overcoming them. Diabetes Obes Metab. 2018;20(3):488-96. https://doi.org/10.1111/dom. 13132. 